Original

\title{
Factores de riesgo ergonómico y su relación con dolor musculoesquelético de columna vertebral: basado en la primera encuesta nacional de condiciones de empleo, equidad, trabajo, salud y calidad de vida de los trabajadores y trabajadoras en Chile (ENETS) 2009-2010
}

\author{
Ergonomic risk factors and their relationship to spinal musculoskeletal pain: \\ based on the first national survey of conditions of employment equity, labour, \\ health and quality of life of the workers in Chile (ENETS) 2009-2010
}

\section{Claudio Muñoz Poblete ${ }^{1,2}$, Jairo Vanegas López ${ }^{2}$ Nella Marchetti Pareto ${ }^{2}$ \\ 1. Departamento Medicina Interna, Facultad de Medicina, Universidad de La Frontera. Chile \\ 2. Escuela Salud Pública, Facultad de Medicina, Universidad de Chile}

Recibido: 28-05-12

Aceptado: 13-06-12

\section{Correspondencia}

Claudio Muñoz Poblete

División Epidemiología

Universidad de Chile. Facultad de Medicina. Escuela de Salud Pública

Independencia 939

Santiago (Chile)

Teléfono 5629786397

Fax 5627377121

E-mail: cfmunoz@med.uchile.cl

Resumen

El dolor de columna constituye un importante problema de salud en la población trabajadora, atribuido a factores individuales y condiciones laborales. Este estudio buscó determinar la influencia de la exposición a factores de riesgo ergonómico durante la jornada laboral sobre la presencia de dolor musculoesquelético en la columna vertebral. Los datos de este estudio se originaron a partir de los resultados que obtuvo la ENETS dirigida a la población laboral chilena. Se pudo concluir que los factores de riesgo ergonómicos que más explican el riesgo de padecer dolor de columna fueron "movimiento repetitivo" (OR: 1.34; CI: 1.08-1.65) seguido de "vibración corporal" (OR: 1.28; CI: 1.04-1.58). En relación al resultado del estudio, es recomendable revisar las políticas públicas en relación a la seguridad y salud laboral así como la legislación vigente relacionada con los problemas musculoesquelético. Estas deben orientarse a la acomodación ergonómica del puesto de trabajo con el propósito de reducir el impacto generado por la exposición reiterada a factores tales como, el manejo de cargas, posturas forzadas y movimientos corporales repetitivos y el efecto acumulativo por trabajos anteriores.

Med Segur Trab (Internet) 2012; 58 (228) 194-204

Palabras claves: Factores riesgo ergonómico, dolor columna vertebral, síntomas musculoesqueléticos, trabajadores.

Abstract

Spinal pain is a major health problem in the working population, attributed to individual factors and conditions factors. This study sought to determine the influence of exposure to ergonomic risk factors during working hours on the presence of musculoskeletal pain in the spine. The data in this study originated from the results obtained ENETS aimed at Chilean workforce. We concluded that ergonomic risk factors that best explain the risk of spinal pain were "repetitive motion" (OR 1.34, CI: 1.08-1.65) followed by "body vibration" (OR: 1.28, CI: 1.04-1.58). Regarding the outcome of the study, you should review the policies in relation to

Factores de riesgo ergonómico y su relación con dolor musculoesquelético de columna vertebral: basado en la primera encuesta nacional de condiciones de empleo, equidad, trabajo, salud y calidad de vida de los trabajadores y trabajadoras en Chile (ENETS) 2009-2010 
occupational health and safety and current legislation related to musculoskeletal problems. These should focus on the accommodation ergonomic workstation in order to reduce the impact caused by repeated exposure to factors such as load handling, awkward postures and repetitive body movements and the cumulative effect from previous work.

Med Segur Trab (Internet) 2012; 58 (228) 194-204

Key words: Risk factors ergonomic, spinal pain, musculoskeletal symptoms, workers. 


\section{INTRODUCCIÓN}

Dentro de los trastornos musculoesqueléticos el dolor de columna representa un importante problema para la Salud Pública no sólo a su alta prevalencia e incidencia sino también debido al alto impacto en la funcionalidad de las personas, en las discapacidades laborales, y en los altos costos económicos asociados al uso de servicios de salud, ausentismo laboral y retiro prematuro ${ }^{1-4}$.

En los países desarrollados este problema de salud ha afectado a los trabajadores en sus años más productivos, considerándose una de las razones más importantes de consulta médica por motivos de discapacidad asociada 5 .

Nuestro país ha experimentado durante las últimas décadas profundos cambios en los diferentes procesos productivos, esto ha traído consigo importantes transformaciones del trabajo y sus riesgos asociados, evidenciándose en la existencia de nuevos riesgos ergonómicos y psicosociales en los ambientes laborales que se asocian a problemas de salud del trabajador ${ }^{6}$.

El dolor de columna representa un complejo problema en su origen y en sus consecuencias, es así como factores individuales, factores ergonómicos del puesto de trabajo y factores psicosociales han mostrado influencia en el desarrollo y persistencia del problema. Estudios epidemiológicos han identificado que estos factores son multidimensionales en su origen y pronóstico ${ }^{7,8}$.

Hay muchas condiciones que pueden provocar dolor de columna causadas principalmente por factores físicos del ambiente laboral y particularmente por ocupaciones que involucran esfuerzo físico 9 . Estudiados desde una perspectiva biomecánica aparecen modelos desarrollados en las últimas dos décadas que nos permiten describir patrones específicos de exposición laboral vinculados con la manipulación de cargas, posturas corporales, movimientos inadecuados y repetitivos sobre la columna vertebral ${ }^{11,12}$.

Dado el desconocimiento existente a la fecha en Chile, tanto de los trabajadores como de los empleadores sobre los riesgos del trabajo y sus daños a la salud, se plantea necesario una aproximación local de las inequidades en salud, de manera de visualizar cómo las desigualdades impactan en la salud y calidad de vida de los trabajadores y trabajadoras.

Con el propósito de recoger información sobre la problemática nacional relacionada con salud ocupacional, Chile desarrolló entre los años 2009 y 2010 la primera Encuesta Nacional de Condiciones de Empleo, Equidad, Trabajo, Salud y Calidad de Vida de los Trabajadores y Trabajadoras en Chile (ENETS), recogiendo entre otras dimensiones, antecedentes sobre las condiciones socio demográficas, laborales, de factores de riesgo ergonómicos y de salud ocupacional, los que constituyen insumos para la realización de este estudio ${ }^{13}$.

Dado lo anterior, este estudio pretende determinar la influencia de la exposición a factores de riesgo ergonómico en la jornada laboral sobre la presencia de dolor musculoesqueletico en la columna considerando la población laboral chilena.

\section{MATERIAL Y MÉTODO}

Entre los años 2009-2010, una muestra de 9.503 trabajadores mayores de 15 años participó de la primera ENETS. Esta muestra fue representativa del total de 7.392.170 trabajadores a nivel nacional. El diseño muestral fue probabilístico, multietápico y estratificado geográficamente por el tamaño de la población urbana y rural del país.

Esta encuesta contiene variables relacionadas a sujetos ocupados y desocupados, información del ingreso, nivel educacional, género y posición socioeconómica de los 
trabajadores; relaciones contractuales de empleo, condiciones cotidianas de trabajo y aspectos asociados a salud y bienestar.

Para el análisis estadístico se uso el software estadístico STATA 11.0 para realizar los análisis descriptivos e inferenciales de la muestra del estudio.

La primera parte del análisis consideró como variable dependiente "dolor de columna", la cual reúne información de dolor de espalda y zona lumbar. Las variables independientes fueron conformadas por los diferentes factores de riesgo ergonómicos y sus tiempos de exposición identificados en el contenido de la encuesta.

La relación entre dolor de columna y las variable independientes fueron estudiadas por medio de una asociación bivariada usando el test de de Chi2. Las variables que obtuvieron una significancia estadística con un valor de $\mathrm{p}<0,05$ fueron incluidas en un modelo de Regresión Logística, donde (0) era la ausencia de dolor de columna y (1) la presencia de dolor de columna. Mediante estrategia de Regresión por eliminación progresiva se fueron eliminando variables hasta conformar un modelo final considerado biomecánicamente plausible y parsimonioso.

Para adecuar la modelación se tomó la decisión de trasformar algunas variables. A partir de los factores psicosociales y de calidad de vida se crearon índices que resumieron los puntajes obtenidos para cada dimensión. Esto permitió establecer una mediana para clasificarlos entre alto y bajo riesgo.

La variable dependiente "dolor de columna" se creó a partir de la fusión de las variables "dolor de espalda" y "dolor en zona lumbar" atribuido al trabajo que realiza actualmente, indicando (0) si los sujetos no tenían dolor en ninguna de estas regiones y (1) si los sujetos encuestados presentaban dolor en al menos una de las dos regiones. Identificándose a un total de 4.093 sujetos de la muestra original con dolor de columna vertebral.

Para mejorar la interpretación del modelo seleccionado, las variables de exposición a factores de riesgo ergonómico ("vibración corporal", "movimiento de alcance", "postura forzada", "manipulación de cargas", "movimiento repetitivo", "trabajo sentado" y "trabajo de pie") se dicotomizaron dejando dos categorías, presencia o ausencia del factor de riesgo, sin considerar los tiempos de exposición.

\section{RESULTADOS}

Del total de 9.503 trabajadores a los que se les preguntó por la presencia de dolor musculoesqueletico en los últimos 12 meses y si este dolor es atribuido al trabajo actual, se observa en la tabla I las zonas corporales de interés para este estudio. Se pudo establecer que un $34 \%$ los trabajadores encuestados reportaron dolor de espalda y un $30.6 \%$ dolor en zona lumbar, presentando las mujeres trabajadoras un mayor reporte en comparación con los hombres.

Tabla I. Dolor músculo esquelético en la columna según sexo

\begin{tabular}{lcccccc}
\hline Zona corporal & \multicolumn{2}{c}{ Hombre (\%) } & \multicolumn{2}{c}{ Mujer (\%) } & \multicolumn{2}{c}{ Total (\%) } \\
\hline & $\begin{array}{c}\text { Últimos 12 } \\
\text { meses }\end{array}$ & $\begin{array}{c}\text { Atribuido al } \\
\text { trabajo }\end{array}$ & $\begin{array}{c}\text { Últimos 12 } \\
\text { meses }\end{array}$ & $\begin{array}{c}\text { Atribuido al } \\
\text { trabajo }\end{array}$ & $\begin{array}{c}\text { Últimos 12 } \\
\text { meses }\end{array}$ & $\begin{array}{c}\text { Atribuido al } \\
\text { trabajo }\end{array}$ \\
\hline Espalda & 31.4 & 82.2 & 38.4 & 75.2 & 34.03 & 79.3 \\
Zona lumbar & 30.3 & 83.6 & 31.2 & 73.4 & 30.6 & 79.7 \\
\hline
\end{tabular}

* Últimos 12 meses: En los últimos 12 meses, ¿Ud. ha tenido dolores permanentes o recurrentes en algunas de las siguientes partes del cuerpo?

* Atribuido al trabajo: ¿Este dolor es, en alguna medida, provocado por el trabajo que realiza? 
En la misma tabla los sujetos que responden que su dolor es atribuido al trabajo actual que realizan se observa un aumento porcentual importante que supera el $73 \%$, siendo mayor para hombres en comparación con las mujeres.

En la tabla II, se muestran los factores de exposición ergonómica reportados en el puesto de trabajo actual, según tiempo de exposición durante la jornada de trabajo (toda la jornada, mitad de la jornada, ocasionalmente, nunca). Los principales factores de riesgo ergonómicos reportados fueron, trabajo de pie (85.4\%), movimiento repetitivo (60.9\%) y postura forzada $(52 \%)$. Otros factores relevantes fueron, el trabajo en posición sentado (50\%), la manipulación de carga (45.9\%), la vibración corporal (33.9\%) y el alcance herramienta $(31.7 \%)$.

Tabla II. Factores exposición ergonómica del puesto trabajo durante la jornada laboral

\begin{tabular}{lcccc}
\hline & Toda jornada & Mitad jornada & Ocasionalmente & Nunca \\
\hline Vibración corporal \% (n: 9452) & 13.9 & 6.2 & 13.8 & 66.1 \\
Alcance herramienta u objetos \% (n: 9427) & 4.9 & 4.4 & 22.4 & 68.4 \\
Postura forzada \% (n: 9451) & 12.9 & 10.2 & 28.9 & 48.1 \\
Manipulación carga \% (n: 9447) & 9.8 & 8.0 & 28.1 & 54.0 \\
Movimiento repetitivo \% (n: 9447) & 20.0 & 12.7 & 28.2 & 39.1 \\
Trabajo sentado \% (n: 9444) & 16.6 & 13.8 & 19.6 & 50.0 \\
Trabajo de pie \% (n: 9458) & 52.6 & 19.7 & 13.1 & 14.5 \\
\hline
\end{tabular}

Haciendo referencia a los tiempos de exposición, en el caso de trabajadores expuestos durante toda la jornada, los factores de riesgo ergonómicos más frecuentes fueron, movimientos repetitivos (20\%), trabajos en posición de pie $(52.6 \%)$ y vibraciones (13.8\%). Continuando en la tabla II, en el caso de trabajadores expuestos durante la mitad de su jornada laboral los factores más reportados fueron, la posición pie (19.7\%), trabajar sentado (13.8\%) y movimientos repetitivos $(12.7 \%)$. Con respecto a la exposición ocasional durante la jornada de trabajo destacan, la postura inadecuada (28.9\%), la manipulación de carga (28.1\%) y el trabajo sentado (28.2\%). Finalmente, en la misma tabla, puede observarse el porcentaje de trabajadores que no reportaron exposición.

En la tabla III, se aprecian variables sociodemográficas de los 4.093 sujetos que reportaron tener dolor de columna, estas características no difieren en mayor medida de lo observado en la muestra original ENETS. Se destaca que está constituida principalmente por hombres (62.4\%), con una media de edad de 43.1 años (SD \pm 13.4). Respecto al estado civil sólo el 46.9\% reportó estar casado. El 33.1\%, declara tener educación básica y el $48.8 \%$ educación media. El $46.3 \%$ posee un nivel de ingresos no mayor a $\$ 180$ mil pesos y el $47.8 \%$ declara tener un ingreso entre $\$ 181$ a $\$ 650$ mil pesos. Con respecto a la antigüedad laboral en el trabajo actual, el $44.5 \%$ tiene más de 5 años y el $27.6 \%$ tiene entre uno y cinco años. Sobre el tipo de previsión de salud, el $80.8 \%$ pertenece al sistema público de salud. Mientras, que un $16 \%$ se encuentra protegido por el sistema salud privado y otros.

La tabla IV muestra el resultado de un análisis de contingencia entre los índices psicosociales y de calidad de vida, dicotomizados en función de su mediana (alto y bajo riesgo) con la nueva variable dolor de columna (presencia o ausencia). Se destaca que la frecuencia entre los factores psicosociales sobre sujetos con o sin dolor, presentan un comportamiento similar entre sí. Por otro lado, los factores psicosociales que resultaron estadísticamente significativos $(\mathrm{p}<0.05)$ fueron "Exigencia psicológica" y Calidad de Vida".

En la tabla V muestra el resultado del test de chi-cuadrado entre el tiempo de exposición a factores de riesgo ergonómicos y la presencia de dolor. Se destaca que los factores de riesgo asociado fueron considerado en su forma original (tiempo exposición durante la jornada). La mayoría de los factores de tiempo de exposición a factores de riesgo ergonómicos muestran tener diferencias significativas $(\mathrm{p}<0,005)$. 
Tabla III. Características los sujetos que presentan dolor de columna*

\begin{tabular}{lll}
\hline Sexo \% (n: 4093) & Hombre & 62.4 \\
Edad, media años \pm DS (n:4092) & & $43.1 \pm 13.4$ \\
Zona \% (n: 4093) & Urbano & 81.7 \\
Estado civil \% (n: 4091) & Casado (a) & 46.9 \\
\hline Educación \% (n: 4086) & Básica & 33.1 \\
& Media & 48.8 \\
& Técnico profesional & 9.2 \\
& Universitaria & 10.2 \\
\hline Ingreso miles de pesos \% (3976) & Hasta 180 & 46.3 \\
& Desde 181 a 650 & 47.8 \\
& Desde 650 & 6.1 \\
\hline Ocupado \% (n: 4093) & & 87.9 \\
\hline Relación de empleo \% (n: 4092) & Estable, seguro en el futuro & 34.8 \\
& Estable, seguro en este momento & 37.9 \\
& Inestable & 27.3 \\
\hline Tiempo de trabajo actual (n: 4092) & Menos de 1 año & 27.9 \\
& 1 a 5 años & 27.6 \\
& 5 o más & 44.5 \\
\hline Afiliación a previsión salud \% (n:4093) & Sistema público FONASA grupo a & 22.8 \\
& Sistema público FONASA otros grupos & 58.4 \\
\hline Dolor de columna considera dolor lumbar y dorsal. & 9.4 \\
\hline
\end{tabular}

Tabla IV. Asociación de entre la exposición a factores psicosociales de alto riesgo y la presencia de dolor

\begin{tabular}{lccc}
\hline & Con dolor & Sin dolor & valor $\mathbf{p}<0.05$ \\
\hline Exigencias psicológicas \% (n:3015) & 83.9 & 16.0 & 0.05 \\
Apoyo social y calidad de liderazgo \% (n:3297) & 83.4 & 16.6 & 0.40 \\
Trabajo activo y desarrollo de habilidades \% (n:3298) & 83.4 & 16.6 & 0.79 \\
Satisfacción con el trabajo \% (n:3005) & 84.5 & 15.5 & 0.08 \\
Calidad de vida \%(n:3326) & 83.7 & 16.3 & 0.03 \\
\hline
\end{tabular}

* Los índices han sido dicotomizados, por debajo la mediana son indicadores de alto riesgo, sobre la mediana bajo riesgo.

Tabla V. Asociación entre la exposición a factor de riesgo del puesto de trabajo y la presencia de dolor de columna

\begin{tabular}{lccccc}
\hline & $\begin{array}{c}\text { Toda } \\
\text { jornada }\end{array}$ & $\begin{array}{c}\text { Mitad } \\
\text { jornada }\end{array}$ & Ocasional & Nunca & valor $\mathbf{p}<\mathbf{0 . 0 5}$ \\
\hline Vibración corporal \% (n: 4156) & 18.7 & 8.2 & 17.4 & 55.7 & 0.001 \\
Alcance herramientas \% (n: 4148) & 6.0 & 5.5 & 25.5 & 62.9 & 0.06 \\
Posturas forzadas \% (n: 4154) & 15.3 & 11.7 & 31.3 & 41.7 & 0.007 \\
Manipulación carga\% (n: 4149) & 12.7 & 10.4 & 30.6 & 46.3 & 0.026 \\
Movimiento repetitivo \% (n: 4154) & 21.6 & 13.9 & 28.2 & 36.3 & 0.081 \\
Trabajo sentado \% (n: 4149) & 16.0 & 13.3 & 18.3 & 52.4 & 0.000 \\
Trabajo de pie \% (n: 4155) & 55.6 & 18.5 & 12.1 & 13.8 & 0.001 \\
\hline
\end{tabular}

En la tabla VI se muestra la exposición a factores de riesgo ergonómico que se identificaron durante el proceso de selección del modelo como variables significativas. La tabla muestra el OR crudo y ajustado, obtenido a través de un modelo de regresión logística. De manera individual los factores que se asociaron a dolor de columna en este estudio fueron "vibración corporal", "postura forzada" y "manipulación de carga", el resto de los factores no mostraron tener significancia estadística. Al construir un modelo 
ajustado, el factor que más explica el riesgo de padecer dolor de columna es "movimiento repetitivo" (OR: 1.34; CI: 1.08-1.65) seguido de "vibración corporal" (OR: 1.28; CI: 1.041.58)).

Tabla VI. Exposición a factores de riesgo ergonómico asociado a Dolor músculo esquelético de columna durante la jornada laboral

\begin{tabular}{lcccc}
\hline & ORcrudo & CI & ORajustado & CI \\
\hline Vibración corporal & 0.90 & $0.82-0.99$ & 1.28 & $1.04-1.58$ \\
Postura forzadas & 1.12 & $1.02-1.23$ & - & - \\
Manipulación carga & 1.13 & $1.02-1.25$ & 0.71 & $0.53-0.94$ \\
Movimiento repetitivo & 0.96 & $0.88-1.04$ & 1.34 & $1.08-1.65$ \\
Trabajo de pie & 1.06 & $0.99-1.13$ & 0.77 & $0.65-0.91$ \\
\hline$* \quad$ Valor $\mathrm{p}<0.05$ & & & & \\
\hline
\end{tabular}

\section{DISCUSIÓN}

Este estudio comprueba la asociación existente entre factores de riesgo ergonómico del trabajo con el dolor de espalda en trabajadores que declararon padecer dolor provocado por el trabajo que realizan. Aunque no todos los factores estudiados mostraron evidencia de esta asociación no le resta relevancia dado que constituye una primera aproximación nacional en el contexto de la ENETS.

Dentro de las limitaciones que podemos mencionar acerca de este estudio es probable que la prevalencia haya sido sobrestimada a la reportada en la literatura ${ }^{13}$ atribuido a la estructura de la encuesta, las definiciones de las dimensiones evaluadas y dado que se consulta por información pasada el sesgo de memoria es más probable. Otras consideraciones son las características socioculturales de la población encuestada, que da cuenta del nivel de salud de la población obrera del país vinculada, principalmente, con trabajos que tienen alta demanda de esfuerzo físico.

En un estudio realizado en Holanda ${ }^{13,14}$ se estimó que la zona musculoesquelética más frecuentemente reportada correspondía a dolor de columna con un $43.9 \%$ (IC 1.6) en el transcurso de los últimos 12 meses y un $21.2 \%$ (IC 1.3) con síntomas de más de tres meses de antigüedad. Los hallazgos de este estudio muestran que el dolor musculo esquelético en columna atribuido a su trabajo supera el $79 \%$. En relación a lo anterior la mayoría de los estudios de base poblacional que se focalizan en el dolor de espalda muestran prevalencias entre $8 \%$ al $82 \%^{15,16}$.

En los trabajadores expuestos, la organización del trabajo afecta los tiempos de exposición laboral a los factores de riesgo ergonómico dado la variedad de funciones que se realizan, en este estudio los sujetos expuestos durante toda la jornada muestran que los factores más frecuentes fueron movimientos repetitivos (20\%), trabajos en posición de pie $(52,6 \%)$ y vibraciones $(13,8 \%)$.

El trabajo físico pesado se ha definido como el trabajo que tiene altas demandas de energía o que requiere de esfuerzo físico para realizarlo. La relación de estas fuerzas con daño en la columna vertebral lo estableció Marras en $1995^{17}$, mediante estudios biomecánicos comprobó que fuerzas de compresión en columna conllevan a daños cuando se supera los niveles de tolerancia de carga de los tejidos. Dado que hasta el momento esta asociación ha sido en términos categóricos brutos, en un nuevo estudio ${ }^{11}$ se pudo distinguir distintos perfiles de exposición dando cuenta que los trabajadores que realizan tareas de distribución están más expuesto a cargas extremas que los empleados de manufactura.

Al transferir objetos de un plano a otro y la frecuencia con que se hace este movimiento, resulta en un aumento de la exigencia física del trabajo. En la actualidad la evidencia no es suficiente para atribuir que el levantamiento de objetos por sí solo sea 
causante de dolor de columna ${ }^{18}$. Como hemos mencionado anteriormente, el trabajo físico pesado ha sido identificado como un factor de riesgo para lesiones en la espalda, no obstante esto no es una regla dado que además existe una amplia gama de resultados en los trabajadores lesionados que inicialmente parecen similares ${ }^{19}$.

Por otro lado, la evidencia también ha demostrado que magnitudes de cargas superiores y repetidas, pueden explicar el deterioro de los discos intervertebrales de la columna, con niveles variables de sintomatología en el individuo, apoyando la existencia de una relación dosis respuesta ${ }^{20}$.

Desde el punto de vista individual la población principalmente afectada son hombres, determinado por el tipo de trabajo relacionado a mayor esfuerzo físico que las mujeres. La edad promedio encontrada corresponde a una población laboral adulta de 43 años, lo cual se condice con el desarrollo de problemas degenerativos en el sistema musculoesquelético.

Sin embargo, la literatura también muestra que se produce un cierto tipo de adaptación más bien tisular a cargas repetidas, lo que podría explicar la adaptación fisiológica del trabajador expuesto a esfuerzo físico durante su vida laboral ${ }^{21}$.

Entre los factores de tipo individual podemos distinguir los demográficos. Los factores demográficos han mostrado ser importantes determinantes de la discapacidad laboral por dolor de columna, los estudios muestran que a mayor edad del trabajador existe un impacto negativo. Los resultados por sexo se mezclan, pero la mayoría de los estudios reportan que las mujeres tienen más probabilidades de verse afectadas negativamente 22,23 . Sin embargo en este estudio son los hombres los que reportan una mayor frecuencia de este problema.

Las características socioeconómicas como el nivel de educación, a pesar de que algunos estudios no muestran evidencia estar asociada a dolor de columna ${ }^{24,25}$, otros estudios $^{26}$, sin embargo, identifican al bajo nivel educacional como un importante predictor de dolor de columna. En nuestro estudio la población está representada principalmente por un sector con un nivel educacional básico con un 33\% y un ingreso que no supera los $\$ 180$ mil pesos en un $46 \%$ de la población afecta por dolor de columna. Al respecto del nivel de ingreso, la evidencia muestra una relación negativa con la presencia del dolor de columna ${ }^{27}$.

En un estudio de seguimiento realizado en empresas industriales ${ }^{28}$ muestra que muy pocos trabajadores están totalmente libres de dolor musculoesquelético y que la transición desde la ausencia de dolor o de menor importancia, a un dolor más severo fue influenciada por factores físicos y psicosociales del lugar de trabajo junto con factores individuales y relacionados con la salud. En este estudio el $87.9 \%$ de la población refiere estar empleada, sólo el 34.8\% refirió sentirse estable en la relación con su empleo y el 44,5\% refirió una antigüedad mayor a 5 años en su empleo actual, lo que podría estar determinando la presencia de dolor de columna.

Los hallazgos en relación a los factores de riesgo psicosociales vinculados al trabajo muestran el $83.9 \%$ de los trabajadores con dolor de columna $(\mathrm{p}<0.05)$ presentan un nivel alto de exigencia psicológica asociada a sus tareas habituales. Sin embargo, evidencia de algunos estudios no es concluyente respecto a su asociación ${ }^{29}$.

Respecto a satisfacción con el trabajo nuestro resultados mostraron que el $84.5 \%$ tenía relación con dolor de columna, sin embargo esto mostro no ser significativo $(\mathrm{p}<0,08)$. Revisiones sistemáticas refieren muestra que no existe asociación entre este factor de riesgo y el dolor de columna ${ }^{30}$. Así mismo, en cuanto a calidad de vida los hallazgos mostraron que los sujetos con dolor de columna tienen una apreciación de la vida bastante negativa, los estudios corroboran estos resultados dando cuenta que existe una relación bidireccional $^{31}$.

La modelación realizada permitió establecer que los sujetos con dolor de columna presentaron una mayor frecuencia de exposición a factores de riesgo ergonómicos como,

Factores de riesgo ergonómico y su relación con dolor musculoesquelético de columna vertebral: basado en la primera encuesta nacional de condiciones de empleo, equidad, trabajo, salud y calidad de vida de los trabajadores y trabajadoras en Chile (ENETS) 2009-2010 
la postura de columna forzada, la manipulación de carga frecuente y trabajar de pie que aquellos trabajadores que no presentaron dolor de columna.

De acuerdo a los modelos conceptuales revisados los determinantes del dolor de columna de origen laboral son multifactoriales, lo cual hace que la tarea de investigación sea sumamente compleja dado que los factores explicativos que intervienen compiten en importancia.

Gran parte de las investigaciones revisadas por National Institute for Occupational Safety and Health ${ }^{32}$ vinculan los problemas de columna con posturas forzadas, en este sentido la flexión de la columna asociada a rotación ha mostrado un OR de 1.97 que implica un mayor riesgo de presencia de dolor de columna en relación a sujetos que no expuestos $^{33}$. También en el acto de manipular carga desde posturas sostenidas (OR 1.96) así como la posición cuclillas y el acto de inclinar la columna (OR 2.01) son reconocidos factores de riesgo de dolor en ella. Con respecto a nuestro estudio, los trabajadores expuestos a uno $\mathrm{u}$ otro riesgo ergonómico de manera individual están asociados claramente con un aumento de la probabilidad de lesión de columna. Sin embargo, al ajustar el modelo, observamos el exponerse simultáneamente a varios factores el riesgo hace la vibración corporal y los movimientos repetitivos representan un mayor riesgo cuando se controla por los demás factores que fueron objeto de análisis de este estudio.

\section{CONCLUSIÓN}

Este trabajo concluyó que la población trabajadora chilena se encuentra expuesta en su lugar de trabajo a múltiples factores de riesgo que conllevan a dolores de columna, al igual que la evidencia revisada, se destacan los factores de riesgo ergonómicos. Complementariamente, permitió visualizar que la cobertura de protección laboral necesariamente debe ampliarse a la población considerada vulnerable, dada sus condiciones educacionales, laborales, contractuales y salariales. Esto debe incluir la revisión de la ley de seguridad en el trabajo existente en Chile, ya que esta deja fuera un número importante de trabajadores informales que tienen niveles similares o superiores de exposición que los trabajadores formales.

Las políticas públicas de seguridad y salud laboral en relación a los problemas musculoesqueléticos, deben necesariamente orientarse a la acomodación ergonómica del puesto de trabajo con el propósito de reducir el impacto generado por la exposición reiterada a factores tales como, el manejo de cargas, posturas forzadas y movimientos corporales repetitivos. Se debe agregar que la ley no contempla el efecto acumulativo producido por trabajos anteriores sobre el sistema musculoesquelético, considerando que actualmente existe una tendencia cada vez mayor que favorece la rotación laboral, dado lo cual se hace recomendable revisar la legislación vigente.

\section{REFERENCIAS BIBLIOGRÁFICAS}

1. Santos AC, Bredemeier M, Rosa KF, Amantea VA, Xavier RM. Impact on the Quality of Life of an Educational Program for the Prevention of Work-Related Musculoskeletal Disorders: a randomized controlled trial. BMC Public Health 2011; 11:60.

2. Woolf AD, Vos T, March L. How to measure the impact of musculoskeletal conditions. Best Pract Res Clin Rheumatol 2010; 24(6):723-32.

3. Nyman T, Grooten WJ, Wiktorin C, Liwing J, Norman L. Sickness absence and concurrent low back and neck-shoulder pain: Results from the MUSIC-Norrtalje study. Eur Spine J. 2007;16:631-3.

4. Katz JN: Lumbar disc disorders and low-back pain: Socioeconomic factors and consequences. J Bone Joint Surg 2006, 88(Suppl 2):21-24.

5. Costa-Black KM, Loisel P, Anema JR, Pransky G. Back pain and work. Best Pract Res Clin Rheumatol $2010 ; 24(2): 227-40$.

Factores de riesgo ergonómico y su relación con dolor musculoesquelético de columna vertebral: basado en la primera encuesta nacional de condiciones de empleo, equidad, trabajo, salud y calidad de vida de los trabajadores y trabajadoras en Chile (ENETS) 2009-2010 
6. Evaluación del Régimen de Accidentes del Trabajo y Enfermedades Profesionales de Chile INFORME FINAL. Facultad de Economía y Negocios. Universidad Alberto Hurtado. Santiago, Febrero 2010.

7. Rubin DI. Epidemiology and risk factors for spine pain. Neurol Clin 2007; 25(2):353-71.

8. Manek NJ, MacGregor AJ. Epidemiology of back disorders: prevalence, risk factors, and prognosis. Curr Opin Rheumatol 2005;17(2):134-40

9. Airaksinen O, Brox JI, Cedraschi C, Hildebrandt J, Klaber-Moffett J, Kovacs F, et al. European guidelines for the management of chronic nonspecific low back pain. Eur Spine J 2006; 15: 192-300.

10. Kumar S. Theories of muskuloskeletal injury causation. Ergonomics. 2001; 44(1):17-47.

11. Marras WS, Lavender SA, Ferguson SA, Splittstoesser RE, Yang G. Quantitative biomechanical workplace exposure measures: distribution centers. J Electromyogr Kinesiol 2010; 20(5):813-22.

12. Ministerio de Salud (MINSAL). Primera Encuesta Nacional de empleo, trabajo, salud y calidad de vida de los trabajadores y trabajadoras de Chile. Informe interinstitucional. Dirección del Trabajo (DT), Instituto de Seguridad Laboral (ISL) ENETS 2009-2010.

13. Diaz-Ledezma C, Urrutia J, Romeo J, Chelen A, Gonzalez-Wilhelm L, Lavarello C. Factors associated with variability in length of sick leave because of acute low back pain in Chile. Spine J 2009; 9 (12):1010-5.

14. Picavet HS SJ. Musculoskeletal pain in the Netherlands: Prevalences, consequences and risk groups, the DMC (3)-study. Pain. 2003(102):167-178.

15. Raspe A, Matthis C, Heon-Klin V, Raspe H. Chronic back pain: more than pain in the back. Findings of a regional survey among insurees of a workers pension insurance fund. Rehabilitation (Stuttg). 2003; 42: 195-203.

16. Andersen JH, Haahr JP, Frost P. Risk factors for more severe regional musculoskeletal symptoms: a twoyear prospective study of a general working population. Arthritis Rheum 2007; 56 (4):1355-64.

17. Marras WS, Lavender SA, Leurgans SE, Fathallah FA, Ferguson SA, Allread WG, et al. Biomechanical risk factors for occupationally related low back disorders. Ergonomics 1995; 38(2):377-410.

18. Wai EK, Roffey DM, Bishop P, Kwon BK, Dagenais S. Causal assessment of occupational lifting and low back pain: results of a systematic review. Spine J 2010; 10(6):554-66.

19. Wasiak R KJ, Pransky G. Work disability and costs caused by recurrence of low back pain: longer and more costly than in first episodes. Spine 2006; 31(2):219-25.

20. Plouvier S, Renahy E, Chastang JF, Bonenfant S, Leclerc A. Biomechanical strains and low back disorders: quantifying the effects of the number of years of exposure on various types of pain. Occup Environ Med 2008; 65(4):268-74.

21. Nordin M, Frankel, V. Basic Biomechanics of the musculoskeletal system. Ed. Lea \& Febigar.2001. 2001

22. Lotters F, Burdorf A. Prognostic factors for duration of sickness absence due to musculoskeletal disorders. Clin J Pain 2006; 22(2):212-21.

23. Agnello A, Brown T, Desroches S, Welling U, Walton D. Can We Identify People at Risk of Non-recovery after Acute Occupational Low Back Pain? Results of a Review and Higher-Order Analysis. Physiother Can 2010; 62(1):9-16.

24. Steenstra IA, Verbeek JH, Heymans MW, Bongers PM. Prognostic factors for duration of sick leave in patients sick listed with acute low back pain: a systematic review of the literature. Occup Environ Med 2005; 62(12):851-60.

25. Fransen M, Woodward M, Norton R, Coggan C, Dawe M, Sheridan N. Risk factors associated with the transition from acute to chronic occupational back pain. Spine. 2002; 27(1):92-8.

26. Alexopoulos EC, Konstantinou EC, Bakoyannis G, Tanagra D, Burdorf A. Risk factors for sickness absence due to low back pain and prognostic factors for return to work in a cohort of shipyard workers. Eur Spine J 2008;17(9):1185-92

27. Krause N DL, Deegan LJ, Rudolph L, Brand RJ. Psychosocial job factors and return-to-work after compensated low back injury: A disability phase-specific analysis. Am J Ind Med. 2001; 40(4):374-92.

28. Andersen JH, Haahr JP, Frost P. Risk factors for more severe regional musculoskeletal symptoms: a twoyear prospective study of a general working population. Arthritis Rheum 2007; 56(4):1355-64. 
29. Hartvigsen J, Lings S, Leboeuf Yde C, Bakketeing L. Psychosocial factors at work in relation to low back pain and consequiences of low back pain; a systematic, critical review of prospective cohort studies. Occup Enviroment Med 2004;61.

30. R A Iles, M Davidson, N F Taylor. Psychosocial predictors of failure to return to work in non-chronic nonspecific low back pain: a systematic review Occup Environ Med 2008; 65:507-517.

31. Kovacs FM, Abraira V, Zamora J, Real MTG, Llobera J, Fernández C, et al. Correlation between pain, disability, and quality of life in patients with common low back pain. Spine 2004; 29(2):206-10.

32. NIOSH. INplSySO. Ergonomic Guidelines for Manual Material Handling. NIOSH Publication. 2007; 131.

33. Sun, J. Z., R. J. Ling, et al. (2011). Risk factors analysis of low back pain among workers in a foundry factory of the automobile company. Chinese Journal of Occupational Medicine. 29(2): 108-11.

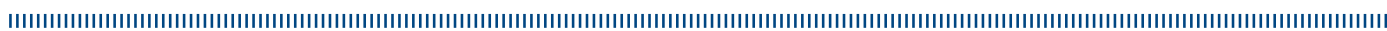

